OAl-PMH: http://www.indteca.com/ojs/index.php/Revista Scientific/oai

Artículo Original / Original Article

\title{
Vínculo entre la programación original de Netflix y la enseñanza universitaria
}

Autor: Rodrigo Urcid Puga Instituto Tecnológico y de Estudios Superiores de Monterrey, TEC

rurcid@tec.mx

Puebla, México

https://orcid.org/0000-0002-5102-3558

\section{Resumen}

El objetivo del siguiente artículo es presentar la relación entre algunas series originales de Netflix y el uso académico que éstas ofrecen. La pregunta de investigación es ¿los programas de esta plataforma pueden servir en el proceso de enseñanza? La teoría de este escrito radica en dos aspectos; primero, la innovación educativa ha tenido una transformación sustancial en los últimos años, y ésta radica en el uso de las Tecnologías de Información y Comunicación (TIC) y, en segundo lugar, el impacto que Netflix tiene entre el estudiantado universitario. La metodología es mixta, de forma cuantitativa se hace el conteo de las series, capítulos, temporadas, géneros, duración, temas abordados y otros elementos que ayudan en cuestiones cuantificables. En materia cualitativa, se utiliza el análisis de contenido y de discurso. En los hallazgos obtenidos se encuentra que el contenido analizado, puede incluirse como herramienta de aprendizaje para diversas áreas de estudio a nivel universitario. Finalmente, los temas que se abordan, la narrativa empleada y las historias que esta plataforma muestra se convierten en herramientas útiles e innovadoras para la didáctica académica.

Palabras clave: educación; innovación; universidad; netflix. educación.

Código de clasificación internacional: 5802.04 - Niveles y temas de

Cómo citar este artículo:

Urcid, R. (2021). Vínculo entre la programación original de Netflix y la enseñanza universitaria. Revista Scientific, 6(22), 311-331, e-ISSN: 2542-2987. Recuperado de: https://doi.org/10.29394/Scientific.issn.2542-2987.2021.6.22.16.311-331

Fecha de Recepción: 20-07-2021
Fecha de Aceptación: 09-10-2021
Fecha de Publicación: 05-11-2021 


\title{
Link Between Netflix Original Programming and College Teaching
}

\begin{abstract}
The objective of the following article is to present the relationship between some Netflix original series and the academic use they offer. The research question is, can the programs of this platform serve in the teaching process? The theory of this writing lies in two aspects; First, educational innovation has undergone a substantial transformation in recent years, and this lies in the use of Information and Communication Technologies (ICT) and, secondly, the impact that Netflix has on university students. The methodology is mixed, quantitatively the counting of series, chapters, seasons, genres, duration, topics addressed and other elements that help in quantifiable issues are made. In qualitative matters, content and discourse analysis is used. In the findings obtained, it is found that the analyzed content can be included as a learning tool for various areas of study at the university level. Finally, the topics that are addressed, the narrative used and the stories that this platform shows become useful and innovative tools for academic didactics.
\end{abstract}

Keywords: education; innovation; college; netflix. education.

International classification code: 5802.04 - Levels and subjects of

How to cite this article:

Urcid, R. (2021). Link Between Netflix Original Programming and College Teaching. Revista

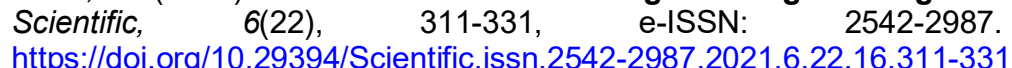

Date Received: 20-07-2021
Date Acceptance:

09-10-2021
Date Publication: 05-11-2021 


\section{Introducción}

Netflix se centra en la industria del entretenimiento y se concentra en exhibir y producir contenidos audiovisuales propios y de terceros; para Gomez y Hunt (2016): el modelo de negocios se basa en utilizar los datos de usuarios para captar y retener suscriptores, establecer fidelidad por medio del servicio y lograr la satisfacción de la clientela con su producto.

En este sentido, Izquierdo-Castillo (2015): señala tres elementos que componen la estrategia de este servicio de streaming: 1). catálogo variado que incluye contenido premium desde su paquete más básico; 2). tarifas que no obligan a estar suscrito permanentemente; y 3 ). servicio orientado a la calidad con sugerencias de contenido y accesibilidad a través de varios dispositivos; además, ofrece contenidos centrados en las decisiones, recomendaciones y hábitos del público consumidor.

En este aspecto, Fernández-Manzano, Neira y Clares-Gavilán (2016): aseguran que Netflix se enfoca en garantizar que el contenido ofertado se consuma y sea interesante; de ahí la rotación del catálogo y cancelación de productos con menor rentabilidad. Bajo este esquema, Lotz (2021): diserta que esta empresa tiene más de 167 millones de suscriptores y que está presente en 190 países; particularmente, México, tiene 4.5 millones, ubicándose en el séptimo lugar de suscriptores a dicha plataforma

Esta empresa se basa en el algoritmo Personal Video Ranker, el cual es una versión más compleja de la clasificación por géneros, la cual es a través de la asignación de metadatos a todo el contenido; y como consecuencia, Madrigal (2014): confirma la existencia de conexiones entre películas con base a patrones como el origen de la producción, año, temas, talento involucrado, popularidad, entre otros.

El concepto de innovación educativa se liga a las TIC; las cuales, según Trejo, Llaven y Culebro (2014): están inmersas en distintos aspectos de la vida diaria, además, contribuyen a la transformación de la sociedad en distintos 
elementos sociales, culturales, económicos, políticos, tecnológicos, etc., tanto de manera grupal como individual.

Los medios digitales se convierten en un estandarte de la educación, y esto se debe a que al alumnado actual se le define como la primera generación de nativos digitales, en otras palabras, son a la vez, quienes más interconectados están, pero también es una generación fuertemente aislada; estas personas tienen su vida documentada y su atención es distributiva, por lo que, Levine y Dean, (2012): mencionan que al tiempo que ven la TV publican en alguna red social, chatean y elaboran un trabajo escolar; de hecho, más del $33 \%$ del estudiantado estadounidense, no está más de diez minutos sin consultar algunos de sus dispositivos.

En ese marco, Scolari (2013): asegura que la utilidad y la temporalidad están vinculados al hecho de consumir contenidos audiovisuales a través de internet, lo cual causa la aparición de los formatos transmedia y consumidores que intervienen en el propio desarrollo de la narrativa, lo cual conlleva una actitud activa.

Una vez dejado en claro los elementos esenciales de esta plataforma, el impacto que los contenidos audiovisuales pueden tener en la formación académica, se puede establecer el objetivo de esta investigación, el cual radica en comprobar que determinadas series de Netflix pueden tener un fin de enseñanza y aprendizaje.

\section{Metodología}

Para tener una investigación sólida y con resultados más contundentes es necesario apelar a métodos académicos de investigación, como los de Hernández, Fernández y Baptista (2014): por ello, para este estudio se recurren a dos enfoques, el cuantitativo y cualitativo ara el primero, se realizó un conteo de las series, temporadas, capítulos, duración, y la calificación que la crítica les otorga. 
El margen bajo el cual se establecieron cuáles series se analizarían, se basa en la calificación que el portal International Movie Database -IMDB- les otorga; en este sentido, se estudiaron los programas que tienen una calificación de ocho o más. Solo se estudiaron las series -ficción- producidas originalmente por Netflix y que comprenden los géneros drama y comedia; se excluyeron películas, programas de entrevistas, especiales, reality shows, documentales, animaciones/caricaturas y docuseries.

En cuanto al estudio cualitativo, se empleó el análisis de contenido, aquí se consideraron las estructuras narrativas y elementos discursivos -guion, temáticas, aspectos técnicos, etc.-, después se creó una lista de temas que los episodios suelen incluir, y se realizó una concentración de las áreas académicas en las cuales puede tener impacto dicho programa.

Los temas designados a cada serie se basaron en la propia narrativa que circunda alrededor de los programas; el análisis minucioso a cada uno de los capítulos y temporadas ayuda a determinar cuáles fueron los tópicos que los productos audiovisuales abordaron. Por otro lado, se realizó una categorización de áreas de conocimiento en las que, de acuerdo con las temáticas, las series pueden apoyar en el proceso de enseñanza.

En total se analizaron 23 programas de corte dramático y trece series de comedia. La suma general de lo estudiado fue de 36 programas, más de 800 episodios y más de ochenta temporadas, la duración de los capítulos oscila desde los noventa hasta los treinta minutos.

\section{Resultados}

Ya que se realiza un estudio mixto, en los siguientes párrafos y tablas, se presentan los hallazgos de acuerdo con la metodología empleada; lo anterior se realiza con el afán de tener un mejor entendimiento de lo estudiado. 


\subsection{Cuantitativo}

La tabla 1, muestra las series analizadas de género dramático; los productos audiovisuales se encuentran listados según el año en que estuvieron disponibles en Netflix.

Tabla 1. Series de género dramático.

\begin{tabular}{|l|c|c|c|c|}
\hline \multicolumn{1}{|c|}{ Título } & $\begin{array}{c}\text { Año de } \\
\text { estreno }\end{array}$ & Temporadas & Episodios & $\begin{array}{c}\text { Calificación } \\
\text { IMDB }\end{array}$ \\
\hline House of Cards & 2013 & 6 & 73 & 8.7 \\
\hline Orange Is the New Black & 2013 & 7 & 91 & 8.1 \\
\hline Marco Polo & 2014 & 2 & 20 & 8 \\
\hline Bloodline & 2015 & 3 & 33 & 8 \\
\hline Daredevil & 2015 & 3 & 39 & 8.6 \\
\hline Sense8 & 2015 & 2 & 24 & 8.3 \\
\hline Narcos & 2015 & 3 & 30 & 8.8 \\
\hline Stranger Things & 2016 & 3 & 25 & 8.8 \\
\hline The Get Down & 2016 & 2 & 11 & 8.3 \\
\hline The Crown & 2016 & 3 & 30 & 8.7 \\
\hline Ozark & 2017 & 3 & 30 & 8.4 \\
\hline Mindhunter & 2017 & 2 & 19 & 8.6 \\
\hline The Punisher & 2017 & 2 & 26 & 8.5 \\
\hline Godless & 2017 & 1 & 7 & 8.3 \\
\hline Altered Carbon & 2018 & 2 & 18 & 8 \\
\hline The Haunt of Hill House & 2018 & 1 & 10 & 8.7 \\
\hline Narcos: Mexico & 2018 & 2 & 20 & 8.4 \\
\hline The Umbrella Academy & 2019 & 2 & 20 & 8 \\
\hline When They See Us & 2019 & 4 & 4 & 8.9 \\
\hline The Dark Crystal: Age of Resistance & 2019 & 1 & 10 & 8.5 \\
\hline The Witcher & 2019 & 1 & 8 & 8.2 \\
\hline Unorthodox & 2020 & 1 & 4 & 8 \\
\hline The Queen's Gambit & 2020 & 1 & 7 & 8.5 \\
\hline Fuente: El Autor & & & & \\
\hline
\end{tabular}

Fuente: El Autor (2021).

En el año 2013, Netflix tiene su primer programa autofinanciado: House of Cards, producto que propone una narrativa basada en la cinematografía, pero trasladada a la TV. Es importante observar que tanto Orange is The New Black como la antes mencionada no tienen más de cuatro temporadas, lo cual habla de historias más precisas y con contenido de mayor calidad en menor tiempo.

Por otra parte, se encuentran las series de comedia, en este sentido, la 
tabla 2 que se presenta, incluye los programas de este género; cabe notar que es hasta el 2015 en que un programa de este género tiene una calificación superior a ocho.

Tabla 2. Series de género comedia.

\begin{tabular}{|l|c|c|c|c|}
\hline \multicolumn{1}{|c|}{ Título } & $\begin{array}{c}\text { Año de } \\
\text { estreno }\end{array}$ & Temporadas & Episodios & $\begin{array}{c}\text { Calificación } \\
\text { IMDB }\end{array}$ \\
\hline Grace and Frankie & 2015 & 5 & 65 & 8.3 \\
\hline Master of None & 2015 & 2 & 20 & 8.3 \\
\hline One Day at a Time & 2017 & 3 & 39 & 8.2 \\
\hline Mystery Science Theater 3000: The Return & 2017 & 2 & 20 & 8 \\
\hline Glow & 2017 & 3 & 30 & 8 \\
\hline Atypical & 2017 & 2 & 18 & 8.3 \\
\hline American Vandal & 2017 & 2 & 16 & 8.2 \\
\hline On My Block & 2018 & 3 & 28 & 8 \\
\hline Kominsky Method & 2018 & 1 & 8 & 8.2 \\
\hline Sex Education & 2019 & 2 & 16 & 8.3 \\
\hline After Life & 2019 & 1 & 6 & 8.4 \\
\hline Dead to me & 2019 & 2 & 20 & 8 \\
\hline Julie and The Phantoms & 2020 & 1 & 9 & 8.4 \\
\hline Fuente: El Autor $(2021)$. & & &
\end{tabular}

Fuente: El Autor (2021).

Las series de comedia tienen características muy peculiares por lo que es importante contextualizar que la narrativa y la trama permiten que se realicen más capítulos por temporada. En este sentido, el programa "Grace and Frankie" -del 2015-, tiene cinco temporadas y más de sesenta episodios hasta el día de hoy, pero también hay productos que, a pesar de ser relativamente nuevos, obtienen gran aceptación y la crítica les otorga una calificación elevada.

Lo anterior no quiere decir que una serie con menos episodios sea mejor que aquella que tiene más temporadas, pero sí es importante mencionar que la narrativa enfocada a historias que pueden ser relatadas en menos capítulos puede tener más impacto en el público meta.

De esta forma quedan abarcados los resultados cuantitativos, los cuales, reflejan que los contenidos de Netflix se caracterizan por ser producciones de pocos episodios y temporadas que no sobrepasan cuatro partes; esto se debe a que la narrativa actual demanda historias más concretas 
y que aborden la temática esencial desde el primer capítulo, y que el desarrollo y la conclusión sean precisas y no se necesiten diez o más temporadas para llegar a la resolución deseada.

\subsection{Cualitativo}

Para tener una mejor idea de los productos analizados, a continuación, se presenta la sinopsis de cada una de las series estudiadas, y los principales discursos que se tienen en las mismas.

Series de drama:

- House of Cards: cuenta la historia de Francis Underwood (Kevin Spacey) y su esposa Claire Underwood (Robin Wright) quienes, mediante engaños, trampas, manipulaciones, y demás estrategias alejadas de la honestidad, buscan poder en la esfera política de Washington, D.C.

- Orange is the new black: cuenta la historia de Piper Chapman (Taylor Schilling), una mujer que es encarcelada por transportar dinero proveniente del narcotráfico y sus vivencias en la cárcel de Litchfield, espacio donde conoce mujeres de diversas razas, preferencias sexuales. La serie se basa en un libro en el que se hace una crítica profunda al sistema penitenciario y a la propia sociedad.

- Marco Polo: cuenta parte de la vida de este, mercader interpretado por Lorenzo Richelmy; dicho personaje realiza un viaje al Oriente con el objetivo de alcanzar esta zona a la cual ninguno de sus predecesores ha podido llegar.

- Bloodline: la historia se desarrolla alrededor de la familia Rayburn y la llegada de uno de los hijos, Danny (Ben Mendelsohn), el cual pone de manifiesto secretos y pasado turbio de dicho linaje.

- Daredevil: relata la vida de Matt Murdock (Charlie Cox), quien de día es 
abogado y de noche es superhéroe; dicho personaje, a pesar de ser ciego, tiene sus otros sentidos desarrollados. Esta serie se basa en el cómic homónimo de Marvel, y es creada por Drew Goddard.

- Sense8: cuenta las aventuras de ocho personas, mismas que se encuentran en un crecimiento mental y conectado por diferentes situaciones que les aquejan.

- Narcos: ahonda el tema del tráfico de cocaína durante los 70's y 80's por todo el mundo. La serie cuenta la guerra entre Pablo Escobar (Wagner Moura) contra el gobierno colombiano -Steve Murphy (Boyd Hobrook) y Pedro Pascal (Javier Peña), agentes de la DEA.

- Stranger Things: programa ambientado en Indiana durante los 80's, y que inicia con la desaparición de un adolescente llamado Will Byers (Noah Schnapp), hecho que desencadena extraños sucesos sobrenaturales, incluida la aparición del personaje "Eleven" (Millie Bobby Brown).

- The Get Down: enfocada en la época de la música disco, R\&B, punk y hip-hop en la década de los setenta. Esta serie gira en torno a la vida de Ezekiel (Justice Smith) y Mylene Cruz (Jerizen Guardiola). Este programa es una crítica al racismo y a la sociedad intolerante.

- The Crown: historia que narra la vida de la Reina Isabel II (Claire Foy, Imelda Staunton y Olivia Colman) de Inglaterra. El programa muestra la vida de la monarquía y la sociedad del Reino Unido.

- Ozark: cuenta la historia de la familia Byrde, quienes se mudan a Missouri y se involucran con un cártel de drogas mexicano y el lavado de dinero que deben hacer para el crimen organizado.

- Mindhunter: basada en la obra de John Douglas y Mark Olshaker; la serie, creada, se centra en los personajes Holden Ford (Jonathan Groff) y Bill Tench (Holt McCallany), quienes crean perfiles psicológicos y 
resuelven crímenes a partir de las entrevistas que les realizan a diversos asesinos y violadores de EUA.

- The Punisher: narra la historia de Frank Castle (Jonathan Bernthal), un soldado estadounidense que tiene como objetivo vengarse de quienes asesinaron a su esposa e hijo; el personaje se basa en un cómic de Marvel.

- Godless: la trama presenta a Frank Griffin (Jeff Daniels), y su grupo de bandidos que van en busca de su excompañero Roy Goode (Jack O'Connell), el cual se esconde en un rancho; lo anterior, desata sucesos que afectan a quienes habitan los distintos pueblos aledaños.

- Altered Carbon: se basa en la novela de Richard K. Morgan. Este programa se desenvuelve en un futuro distópico que explora lo que pasaría cuando la mente humana puede ser "guardada" digitalmente y descargada en otro cuerpo.

- The Haunting of Hill House: creada por Mike Flanagan y basada en el libro de Shirley Jackson, sigue la historia de Steven Crain (Michiel Huisman) y Olivia Crain (Carla Gugino), y cómo fue su vida en la mansión embrujada.

- Narcos México: la serie presenta el mundo del narcotráfico mexicano desde los años ochenta. La trama se centra en Miguel Ángel Félix Gallardo (Diego Luna), líder del cártel y Kiki Cameron (Michael Peña) agente -encubierto- de la DEA.

- The Umbrella Academy: esta serie es una adaptación a partir del cómic de Gerard Arthur Way. La narrativa se enfoca en una familia -un tanto fuera de lo normal- de superhéroes que investiga la muerte de su padre.

- When They See Us: serie que narra el caso de cinco jóvenes afroamericanos que son condenados injustamente a prisión por supuestos casos violación a una mujer en Nueva York. 
- The Witcher: se desprende de la saga literaria creada por Andrzej Sapkowski. La narrativa se centra en Geralt de Rivia (Henry Calvill) y sus vivencias al descubrir a una hechicera y a una princesa las cuales guardan un secreto.

- Unorthodox: serie que relata la vida de Esther Shapiro (Shira Haas), quien es parte del Judaísmo Jasídico. La joven, de acuerdo con las costumbres, debe casarse con el rabino Yanki, suceso que la obliga a huir de la comunidad y mudarse a Alemania para reencontrarse con su madre.

- The Queen's Gambit: serie que se enfoca en la vida de Beth Harmon (Anya Taylor-Joy) y el virtuosismo que tiene para el ajedrez. Durante su adolescencia y juventud, vive una serie de situaciones que le forjan un carácter muy particular

Series de comedia:

- Grace \& Frankie: cuenta la vida de dos amigas, Grace Hanson (Jane Fonda), y Frankie (Lily Tomlin), las cuales descubren que sus respectivos esposos llevan más de veinte años en una relación amorosa y anuncian que van a vivir juntos.

- Master of None: narra ciertos momentos de Dev (Aziz Ansari), el cual tiene 32 años y busca éxito en el mundo del espectáculo. La trama se centra en diversas situaciones cotidianas como citas casuales, eventos familiares, ambiente de las celebridades, y otras experiencias.

- One Day at a Time: remake del sitcom de 1975. La historia narra la vida de una familia cubanoamericana; pero se centra en Penélope Álvarez (Justina Machado), la cual es una exmilitar, está recién divorciada y tiene una hija adolescente.

- Mystery Science Theater 3000 - The return: narra la historia de Jonah 
Heston (Jonah Ray) y sus robots, que quedan atrapados en el "satélite del amor", aquí Max (Patton Oswalt) y Kinga (Felicia Day), les obligan a ver las peores películas "serie B" de ciencia ficción.

- Glow: programa inspirado en la historia de la liga de lucha libre femenina, conocida como: Gorgeous Ladies of Wrestling G.L.O.W., en la década de los 80's en EUA. La historia se enfoca en las acciones de una actriz desempleada, Ruth Wilder (Alison Brie) y sus vivencias para unirse a este grupo.

- Atypical: se enfoca en Sam (Keir Gilchrist), preparatoriano de 18 con autismo. El personaje busca una vida independiente sin la ayuda de su familia

- American Vandal: falso documental que narra el caso de Dylan Maxwell (Jimmy Trato), adolescente que es acusado de hacer grafitis con símbolos fálicos en los coches de la escuela en la cual estudia; sin embargo, él se declara inocente, y para probarlo un joven cineasta se pone de su lado y crea un documental que ayuda a probar su inocencia.

- On my Block: serie que relata la historia de César (Diego Tinoco), Ruby (Jason Genao), Monse (Sierra Capri) y Jamal (Brett Gray), jóvenes estadounidenses que buscan sobrellevar la amistad, vida amorosa, experiencias familiares y demás aspectos de la juventud.

- The Kominsky Method: narra parte de la vida de Sandy Kominsky (Michael Douglas), un actor retirado y que ahora, se dedica a dar clases de actuación. La serie describe las vivencias de dicho personaje y de su amigo Norman (Alan Arkin), dos adultos de la tercera edad que deben enfrentar las decisiones que han tomado a lo largo de los años.

- Sex Education: Otis (Asa Butterfield), ofrece consejos sobre sexualidad a partir de los conocimientos que ha adquirido de su mamá quien es sexóloga. Él, y su amiga Maeve (Emma Mackey), deciden crear una 
clínica "clandestina" y brindar asesoría en estos temas.

- After Life: programa con características de comedia oscura que narra la historia de Tony (Ricky Gervais), reportero que, a partir de la muerte de su esposa, tiene actitudes negativas, impulsivas y rebeldes para con la gente que le rodea.

- Dead to me: narra la relación entre Jen (Christina Applegate) y Judy (Linda Cardellini). Jen, acaba de perder a su esposo, y tras este suceso, se entabla una extraña amistad entre ambas mujeres.

- Julie and the phantoms: se enfoca en la joven Julie (Madison Reyes), una estudiante que pierde el gusto por la música cuando muere su madre; sin embargo, esto cambia cuando en el estudio de música de su casa, aparecen fantasmas que son músicos, con los cuales crea una nueva banda.

Ya se tiene la sinopsis de las series, para complementar lo anterior y a partir del análisis de contenido y de discurso elaborado a los episodios, se construye una tabla en la que se incluyen los temas que los programas abordan; para ello, se asigna un número a cada tema. Cabe notar que el número es un referente para que se tenga mayor claridad en los párrafos donde se describe cómo puede involucrarse la serie con ciertos temas de aprendizaje.

Tabla 3. Número de categoría y temas.

\begin{tabular}{|c|c|}
\hline Categoría & Tema \\
\hline 1 & Amistad \\
\hline 2 & Amor/Desamor \\
\hline 3 & Comunicación humana \\
\hline 4 & Comunidad LGBTQ+ \\
\hline 5 & Corrupción \\
\hline 6 & Crimen \\
\hline 7 & Dinero \\
\hline 8 & Espionaje \\
\hline 9 & Familia \\
\hline 10 & Gobierno \\
\hline
\end{tabular}




\begin{tabular}{|c|c|}
\hline 11 & Historia \\
\hline 12 & Lealtad \\
\hline 13 & Leyes / Justicia \\
\hline 14 & Liderazgo \\
\hline 15 & Machismo/Feminismo \\
\hline 16 & Matrimonio / relaciones de pareja \\
\hline 17 & Medios Masivos \\
\hline 18 & Música \\
\hline 19 & Narcotráfico \\
\hline 20 & Poder \\
\hline 21 & Racismo \\
\hline 22 & Relaciones y comportamiento humano \\
\hline 23 & Religión \\
\hline 24 & Represión (social y/o sexual) \\
\hline 25 & Sistema penitenciario \\
\hline
\end{tabular}

Fuente: El Autor (2021).

Como puede observarse en la tabla 3, en total se identificaron 25 temas distintos que se contemplan en diversos episodios de todas las series analizadas; es importante acotar que estos tópicos "generalizan" muchas de las narrativas encontradas en los programas, y se decide enunciarlos a partir no sólo del análisis de contenido, sino de estudios previos a los cuales se recurre.

Una vez especificados los temas que las distintas series abordan, es posible identificar las áreas de conocimiento que pueden verse beneficiadas si se crea el vínculo entre los episodios y el proceso de aprendizaje; cabe señalar que se llega a éstas, a partir de un análisis profundo a diversas Instituciones de Educación Superior (IES) que se encuentran en diversos rankings. Las áreas de conocimiento quedan integradas de la siguiente forma: a). Arquitectura-diseño; b). Artes; c). Ciencias exactas; d). Ciencias sociales; e). Comunicación y lenguaje; f). Administrativo-económicas; g). Educación; h). Estudios ambientales; i). Estudios creativos; j). Filosofía y humanidades; k). Ingenierías; I). Innovación; m). Salud-medicina; y n). Tecnologías de información

En total se tienen catorce grandes áreas de conocimiento, en las cuales, 
las series pueden encontrar una correlación para complementar el aprendizaje a nivel educativo superior. El contenido que se oferta en los programas puede ser aplicado como "estudios de caso" y encontrar una similitud con cuestiones de la vida cotidiana. En las siguientes tablas se muestra el nombre de la serie, la clasificación de esta, los temas que usualmente abordan y las áreas de conocimiento que pudieran verse impactadas.

Tabla 5. Series de comedia con temáticas y áreas de estudio en las que pueden ser aplicadas.

\begin{tabular}{|c|c|c|}
\hline Título & Temas abordados & Áreas de aplicación \\
\hline Grace and Frankie & $\begin{array}{c}1,2,3,4,9,12,14,15,16 \\
22,24\end{array}$ & $B, D, E, G, J, M$ \\
\hline Master of None & $\begin{array}{c}1,2,3,4,9,12,14,15,16 \\
17,21,22,24\end{array}$ & $A, B, D, E, I, J, N$ \\
\hline One Day at a Time & $1,2,9,12,14,15,16,22$ & $\mathrm{D}, \mathrm{E}, \mathrm{F}, \mathrm{G}, \mathrm{J}$ \\
\hline $\begin{array}{l}\text { Mystery Science Theater 3000: The } \\
\text { Return }\end{array}$ & $1,3,8,9,12,14,22$ & $C, D, E, G, I, J, M, N$ \\
\hline 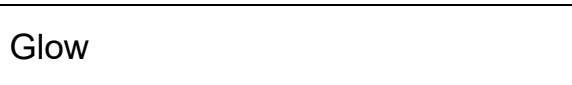 & $\begin{array}{c}1,2,3,4,9,11,12,14,15 \\
16,17,20,22\end{array}$ & $\begin{array}{c}\text { A, B, D, E, F, G, I, J, } \\
L, M\end{array}$ \\
\hline Atypical & $\begin{array}{c}1,2,3,4,9,12,14,16,22, \\
24\end{array}$ & $\begin{array}{c}\mathrm{B}, \mathrm{D}, \mathrm{E}, \mathrm{F}, \mathrm{G}, \mathrm{H}, \mathrm{J}, \mathrm{L}, \\
\mathrm{M}, \mathrm{N}\end{array}$ \\
\hline American Vandal & $\begin{array}{c}1,3,5,6,8,9,12,14,17 \\
22\end{array}$ & $\mathrm{D}, \mathrm{E}, \mathrm{G}, \mathrm{H}, \mathrm{I}, \mathrm{J}, \mathrm{K}, \mathrm{L}$ \\
\hline On My Block & $\begin{array}{c}1,2,3,4,9,12,14,15,16, \\
21,22\end{array}$ & $A, B, D, E, F, G, I, J$ \\
\hline Kominsky Method & $1,2,3,9,12,16,22$ & $\mathrm{~B}, \mathrm{D}, \mathrm{E}, \mathrm{F}, \mathrm{G}, \mathrm{I}, \mathrm{J}, \mathrm{M}$ \\
\hline Sex Education & $\begin{array}{c}1,2,3,4,7,9,12,14,15 \\
16,20,21,22,24\end{array}$ & B, C, E, F, G, J, L, M, \\
\hline After Life & $1,2,3,9,12,15,16,17,22$ & $\mathrm{D}, \mathrm{E}, \mathrm{F}, \mathrm{G}, \mathrm{I}, \mathrm{J}, \mathrm{M}$ \\
\hline Dead to me & $\begin{array}{c}1,2,6,8,9,12,13,15,16, \\
22,\end{array}$ & $\begin{array}{c}A, C, D, E, F, G, H, J, \\
K, L, M\end{array}$ \\
\hline Julie and the Phantoms & $1,2,3,9,12,14,16,18,22$ & $\mathrm{~B}, \mathrm{C}, \mathrm{D}, \mathrm{E}, \mathrm{G}, \mathrm{I}, \mathrm{J}, \mathrm{M}$ \\
\hline
\end{tabular}

Fuente: El Autor (2021).

La tabla 5, refleja que las series dramáticas tienen un alto contenido de temas que van desde lo económico, hasta el educativo, la filosofía, la ingeniería y demás áreas que pueden consolidar el aprendizaje. Además, es interesante observar que prácticamente todos los programas abarcan todos los tópicos a los cuales se hace referencia, y no solo eso, sino que, de acuerdo 
con lo estudiado, se puede comprobar que los programas originales de Netflix que tienen calificación de ocho o superior pueden ayudar al aprendizaje.

Ahora bien, debe señalarse que son series que pertenecen al género de comedia y que a pesar de que la narrativa pudiera ser más simple, la plataforma de streaming, opta por crear contenidos con tópicos actuales, de crítica social y esto hace que lleguen a diferentes audiencias.

El que estos programas sean capaces de abordar distintos contenidos habla de un público que busca series con historias más completas y que tengan una finalidad más allá del entretenimiento. Y, prácticamente todos los estudios de pregrado pueden verse impactados si se utiliza la programación de forma idónea. En cuanto a las series dramáticas, la tabla 6 queda conformada de la siguiente forma:

Tabla 6. Series dramáticas con temáticas y áreas de estudio en las que pueden ser aplicadas.

\begin{tabular}{|l|c|c|}
\hline \multicolumn{1}{|c|}{ Título } & Temas abordados & Áreas de aplicación \\
\hline House of Cards & $\begin{array}{r}3,5,6,7,8,10,12,13,14,16, \\
20,22,25\end{array}$ & C, D, E, F, G, H, I, J, K, L, M, N \\
\hline $\begin{array}{l}\text { Orange Is the New } \\
\text { Black }\end{array}$ & $\begin{array}{r}1,2,3,4,5,6,7,8,9,12,13, \\
14,15,16,19,20,21,22,24, \\
25\end{array}$ & A, C, D, E, F, G, H, J, K, M \\
\hline Marco Polo & $\begin{array}{r}1,3,6,7,8,9,10,11,12,14, \\
20,22\end{array}$ & A, B, C, D, E, F, G, J, K, L, M \\
\hline Bloodline & $\begin{array}{r}1,3,5,6,7,9,12,13,16,20, \\
22,25\end{array}$ & A, D, E, F, G, J, M \\
\hline Daredevil & $\begin{array}{r}1,2,3,5,6,7,8,10,12,13,14, \\
19,20,22,25\end{array}$ & C, D, E, F, J, K, L, M \\
\hline Sense8 & $\begin{array}{r}1,2,3,4,5,6,8,9,12,14,15, \\
16,18,19,20,21,22,23,24\end{array}$ & A, B, C, D, E, G, J, L, M, N \\
\hline Narcos & $\begin{array}{r}1,2,3,5,6,7,8,9,10,11,12, \\
13,14,16,17,19,20,22,25\end{array}$ & C, D, E, F, H, I, J, K, L, M, N \\
\hline Stranger Things & $\begin{array}{r}1,2,3,8,9,10,11,12,14,16, \\
17,20,22\end{array}$ & B, C, D, E, G, H, I, K, M, N \\
\hline The Get Down & $\begin{array}{r}1,2,3,6,7,9,10,11,12,13, \\
14,16,17,18,19,21,22,24\end{array}$ & A, B, D, E, G, I, J, L, N \\
\hline The Crown & $\begin{array}{r}1,2,3,5,6,7,8,9,10,11,12, \\
13,14,16,20,22\end{array}$ & A, B, D, E, F, G, J, K, M \\
\hline Ozark & $\begin{array}{r}1,2,3,5,6,7,8,9,10,12,13, \\
14,16,19,20,22,\end{array}$ & A, D, E, F, G, H, J. K, L, \\
\hline
\end{tabular}


Instituto Internacional de Investigación y Desarrollo Tecnológico Educativo INDTEC, C.A.

DOI: https://doi.org/10.29394/Scientific.issn.2542-2987.2021.6.22.16.311-331

OAI-PMH: http://www.indteca.com/ojs/index.php/Revista Scientific/oai

Artículo Original / Original Article

\begin{tabular}{|c|c|c|}
\hline Mindhunter & $\begin{array}{c}1,3,6,8,9,10,11,12,13,14 \\
16,20,22\end{array}$ & $\mathrm{C}, \mathrm{D}, \mathrm{E}, \mathrm{G}, \mathrm{J}, \mathrm{M}, \mathrm{N}$ \\
\hline The Punisher & $\begin{array}{c}1,3,5,6,7,8,9,10,12,13,14 \\
19,20,22\end{array}$ & $\mathrm{C}, \mathrm{D}, \mathrm{F}, \mathrm{H}, \mathrm{J}, \mathrm{K}, \mathrm{M}, \mathrm{N}$ \\
\hline Godless & $\begin{array}{c}1,2,3,6,7,9,11,12,13,14 \\
20,22\end{array}$ & $\mathrm{~A}, \mathrm{C}, \mathrm{D}, \mathrm{F}, \mathrm{H}, \mathrm{J}, \mathrm{K}, \mathrm{M}$ \\
\hline Altered Carbon & $\begin{array}{c}1,2,3,5,6,8,10,12,14,16 \\
20,22\end{array}$ & $A, C, D, E, F, H, J, K, L, M, N$ \\
\hline $\begin{array}{l}\text { The Haunting of Hill } \\
\text { House }\end{array}$ & $1,3,8,9,12,14,16,20,22$ & $\mathrm{~A}, \mathrm{C}, \mathrm{D}, \mathrm{E}, \mathrm{G}, \mathrm{H}, \mathrm{J}, \mathrm{M}$ \\
\hline Narcos: Mexico & $\begin{array}{c}1,2,3,5,6,7,8,9,10,11,12 \\
13,14,16,17,19,20,22,25\end{array}$ & $\mathrm{C}, \mathrm{D}, \mathrm{E}, \mathrm{F}, \mathrm{H}, \mathrm{I}, \mathrm{J}, \mathrm{K}, \mathrm{L}, \mathrm{M}, \mathrm{N}$ \\
\hline $\begin{array}{l}\text { The Umbrella } \\
\text { Academy }\end{array}$ & $\begin{array}{c}1,2,3,4,6,8,9,10,11,12,14 \\
15,16,20,22,24\end{array}$ & $A, B, C, D, E, G, J, L, N$ \\
\hline When They See Us & $\begin{array}{c}1,3,5,6,9,10,11,12,13,14 \\
17,20,21,22,24,25\end{array}$ & $\mathrm{C}, \mathrm{D}, \mathrm{E}, \mathrm{G}, \mathrm{I}, \mathrm{J}, \mathrm{M}$ \\
\hline The Witcher & $\begin{array}{c}1,2,3,5,6,8,9,11,12,14,16 \\
20,22\end{array}$ & $A, C, D, E, F, H, J, M$ \\
\hline Unorthodox & $\begin{array}{c}1,2,9,11,12,14,15,16,22 \\
23,24\end{array}$ & $B, D, E, F, G, I, J, M$ \\
\hline $\begin{array}{l}\text { The Queen's } \\
\text { Gambit }\end{array}$ & $1,2,3,9,11,14,15,22$ & $\mathrm{C}, \mathrm{D}, \mathrm{E}, \mathrm{J}, \mathrm{M}$ \\
\hline
\end{tabular}

Fuente: El Autor (2021).

Los programas de corte dramático, al presentar una narrativa más compleja y completa, tienen mayor impacto en distintas áreas del conocimiento. Como se puede observar, todos los temas a los cuales se hace mención son abordados por todas las series, y, en este sentido, se puede decir que los productos audiovisuales conjugan una serie de narrativas que soportan un contenido de calidad y de historias que pueden aportar de forma considerable a distintas áreas académicas.

Por otro lado, es interesante que prácticamente todas las carreras que la mayoría de las IES con calidad académica y evaluadas por distintos organismos pueden obtener grandes beneficios de los contenidos originales que Netflix ofrece, lo importante, es saber aplicarlos y entender que no importa si se estudia actuaría, música, ingeniería civil, derecho, emprendimiento, enfermería, pedagogía, biología o teología, los programas pueden aportar 
mucho, sobre todo, en una época en la que el aprendizaje se apoya en el material digital.

Cualquiera de los productos seleccionados puede ser de gran ayuda para desarrollar el aprendizaje en las áreas de análisis y producción de contenidos audiovisuales, de hecho, hay diversas narrativas y elementos técnicos que pueden ser estudiados.

Lo anterior debe ser considerado como un punto a favor de las TIC, donde su uso puede ser explotado, y así lograr que la enseñanza se acerque a productos audiovisuales de alta calidad y gran narrativa; mismos que, de una u otra forma, pueden tener impacto en la educación universitaria; por ello, la TV, el streaming y el uso de tecnología puede vincularse con el aprendizaje y fortalecer la multi, trans e interdisciplinariedad.

Finalmente, se puede notar que las series televisivas seleccionadas, según su discurso, pueden ser de gran ayuda para distintos planes de estudio a nivel pregrado; sin embargo, es también importante señalar que, para lograr un mejor impacto, lo ideal es que el profesorado logre una inmersión profunda en duchos contenidos y así lograr mejores resultados.

\section{Conclusiones}

Netflix puede convertirse en una herramienta de apoyo a la educación y no solo concebirse como una plataforma de entretenimiento; esto se debe a que, en promedio, el estudiantado que cursa la universidad está un promedio de nueve horas al día interactuando con algún dispositivo electrónico por ello, utilizar los programas previamente analizados, puede ser una práctica para incorporar el uso de las TIC a la educación.

Lo que se debe considerar es que el profesorado tenga contacto con los contenidos audiovisuales que Netflix ofrece y conozca la forma de integrarlas a la dinámica de las clases; además, no hay que perder de vista que el alumnado está en constante contacto con dicha plataforma y la mejor 
manera de integrar ambos elementos, es acercar el proceso educativo con el entretenimiento.

Así pues, los contenidos de Netflix ofrecen una serie de narrativas que bien pueden vincularse con el entorno académico y que, de ser utilizadas de forma adecuada, pueden ser un vínculo importante para los procesos de educación y aprendizaje; las historias, los personajes y las temáticas sirven para que el alumnado aumente su conocimiento sobre algunos temas y por qué no, el profesorado tenga sesiones más dinámicas y menos pasivas.

Cabe notar que por el momento son escasos los estudios que relacionan el aprendizaje con la exposición a programas ficticios de Netflix, por ello, este texto se considera que puede tener un aporte en materia de innovación educativa.

Como futuros estudios, este análisis se puede ver complementado con análisis de contenido a un programa o serie en específico, y no solo de Netflix, sino de otras plataformas. También se pueden realizar estudios cualitativos y cuantitativos entre quienes buscan conocimiento en esta clase de productos televisivos y cuáles son los efectos y alcances que tienen entre los consumidores.

Finalmente, se puede comprobar el objetivo planteado al inicio de esta investigación, es decir, esta plataforma crea productos de gran calidad audiovisual y gracias a los tipos de historias que desarrolla, no solo se está ante contenidos de entretenimiento, sino que el profesorado puede encontrar grandes aportaciones y conocimiento sobre distintas disciplinas.

\section{Referencias}

Fernández-Manzano, E., Neira, E., \& Clares-Gavilán, J. (2016). Data management in audiovisual business: Netflix as a case study. El profesional de la información, 25(4), 568-576, e-ISSN: 1699-2407. Recovered from: https://doi.org/10.3145/epi.2016.jul.06 
Gomez-Uribe, C., \& Hunt, N. (2016). The Netflix Recommender System: Algorithms, Business Value, and Innovation. ACM Transactions on Management Information Systems, 6(4), 13-19, e-ISSN: 2158-6578.

Recovered from: https://doi.org/10.1145/2843948

Hernández, R., Fernández, C., \& Baptista, M. (2014). Metodología de la Investigación. Sexta edición, ISBN: 978-1-4562-2396-0. México:

McGraw-Hill / Interamericana Editores, S.A. de C.V.

Izquierdo-Castillo, J. (2015). El nuevo negocio mediático liderado por Netflix: estudio del modelo y proyección en el mercado español. El profesional de la información, 24(6), 819-826, e-ISSN: 1699-2407. Recuperado de:

http://profesionaldelainformacion.com/contenidos/2015/nov/14.html

Levine, A., \& Dean, D. (2012). Generation on a Tightrope: A Portrait of Today's College Student. ISBN: 978-0470376294. Inglaterra: JosseyBass.

Lotz, A. (2021). In between the global and the local: Mapping the geographies of Netflix as a multinational service. International Journal of Cultural Studies, 24(2), 195-215, e-ISSN: 1367-8779. Recovered from: https://doi.org/10.1177/1367877920953166

Madrigal, A. (2014). How Netflix Reverse-Engineered Hollywood. United Kingdom, European: The Atlantic Monthly Group, LLC.

Scolari, C. (2013). Narrativas Transmedia: Cuando Todos los Medios Cuentan. España: Deusto Ediciones.

Trejo, M., Llaven, G., \& Culebro, M. (2014). Retos y Desafíos de las TIC y la Innovación Educativa. Atenas, 4(28), 130-143, e-ISSN: 1682-2749. Recuperado de: https://www.redalyc.org/articulo.oa?id=478047204011 
